\title{
BEAM COUPLING IMPEDANCE SIMULATIONS AND MEASUREMENTS FOR THE LHC FP420 DETECTOR
}

\author{
F. Roncarolo *, R. Appleby, R.M. Jones
}

The Cockcroft Institute, Daresbury, Warrington, WA4 4AD U.K. Department of Physics and Astronomy, The University of Manchester, Oxford Road, Manchester, M13 9PL, United Kingdom 


\title{
BEAM COUPLING IMPEDANCE SIMULATIONS AND MEASUREMENTS FOR THE LHC FP420 DETECTOR
}

\author{
F. Roncarolo*, R. Appleby, R.M. Jones \\ The Cockcroft Institute, Daresbury, Warrington, WA4 4AD U.K. \\ Department of Physics and Astronomy, The University of Manchester, \\ Oxford Road, Manchester, M13 9PL, United Kingdom
}

\section{Abstract}

The FP420 collaboration [1] aims at designing forward proton tagging detectors to be installed in the LHC sectors 420 meters downstream of the ATLAS and CMS detectors. The experiment increases the impedance of the machine, because it requires an indentation of the beam pipe in the form of a pocket to permit the detector close access to the beam. We investigate the geometric and resistive wall impedance with both numerical simulations and a purpose built experiment.

\section{INTRODUCTION}

The electromagnetic interaction between the beam and its surroundings constitutes the wake-field created by the beam. This wake-field has both geometrical and resistive wall components. In practise it is convenient to characterise the wake-field in terms of its Fourier transform, known as the coupling impedance. The wake field short range and long range components can result in single and multi-bunch instabilities leading to beam losses and dilution of the beam emittance.

The relevant upper frequency limit is assessed by the nominal LHC beam bunch length, $\sigma_{z}=0.25 \mathrm{~ns}$ (r.m.s.). This permits us to limit our study up to a frequency of $3 \mathrm{GHz}$.

The proposed FP420 experiment foresees 4 detectors, each composed of 2 stations. The station design used for the impedance studies is shown in Fig. 1 and consists of a 1 meter long beam pipe with a $60 \mathrm{~cm}$ long "pocket". A second design proposal subdivides the station in two (30 and $20 \mathrm{~cm}$ long) pockets separated of $20 \mathrm{~cm}$. The majority of the results presented in this paper are expressed

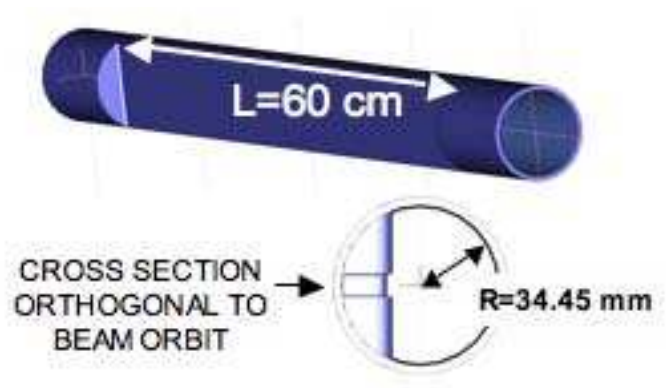

Figure 1: FP420 single pocket station, as designed for numerical simulations.

*federico.roncarolo@cern.ch

05 Beam Dynamics and Electromagnetic Fields in terms of effective longitudinal impedance: $\left(Z_{L} / n\right)=$ $Z_{L}(f) /\left(f / f_{0}\right)$, where $f_{0}=11 k H z$ is the beam revolution frequency in the LHC. All the calculations and measurements refer to a FP420 station made of stainless steel.

\section{COAXIAL WIRE METHOD}

The coaxial wire measurement setup [2] consists of a thin wire that is stretched inside the Device Under Test (DUT) along the reference beam trajectory. The wire is electrically connected to a Vector Network Analyzer (VNA) that acts as RF source and signal processing device. A typical problem of this method concerns the matching between the RF source and the DUT. After demonstrating with numerical simulations the poor effectiveness of tapering cones in the LHC beam spectrum frequency range, we introduced the use of matching resistors, as described in [3]. Even though the resistors limit the region in which the DUT is matched to the generator, the further complication of using the matching cones has a limited practical benefit.

The deviation of the impedance of the DUT from that of the reference vessel can be modeled with a loaded transmission line [4]. Solving the resulting non-linear equation to first order in impedance enables an explicit relation between the longitudinal impedance and S21 to be obtained. This is referred to as the "log" formula ${ }^{1}$ [2]:

$$
Z_{L}(\omega)=-2 Z_{c} \ln \frac{S_{21}^{D U T}(\omega)}{S_{21}^{R E F}(\omega)}
$$

where $Z_{c}$ is the characteristic line impedance.

\section{SIMULATIONS}

The results of three different numerical simulation packages are presented in this paper. Ansoft HFSS ${ }^{(C)}$ [5] was used to simulate the stretched wire setup and calculate the longitudinal impedance according to Eq. (1), while CST Particle Studio $^{\circledR}$ (PST)[6] and GDFIDL [7] provide a direct calculation of the electromagnetic field induced by a passing bunch on the surrounding structure.

The real part of the effective longitudinal impedance, calculated with the three different codes, is shown in Fig. 2. For the single pocket geometry, four narrow band impedance peaks result both from HFSS and PST between

\footnotetext{
${ }^{1}$ The use of the "improved log-formula" gives very similar results for the studies presented here.
}

D04 Instabilities - Processes, Impedances, Countermeasures 


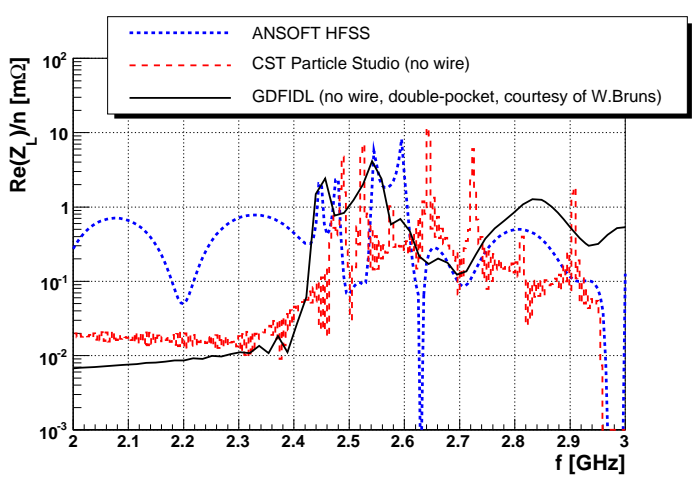

Figure 2: Real part of the effective longitudinal impedance simulated with three different codes, with the wire at $3 \mathrm{~mm}$ from the pocket wall.

2.4 and $2.75 \mathrm{GHz}$. The frequency difference is attributed to the presence of the wire in the HFSS simulation. Two of the four resonances are significantly reduced for the double pocket simulation with GDFIDL. The resonances that we observe (in both HFSS simulations and experiments.) for $f<2.4 \mathrm{GHz}$ are understood to be an artefact of the wire and they do not represent a beam impedance effect. In performing these simulations, a beta-version of PST has been used. Additional simulations of the double pocket geometry with HFSS and PST are in progress.

\section{Transverse Impedance}

For frequencies below $2 \mathrm{GHz}$, the transverse impedance introduced by the FP420 insertion is dominated by the resistive wall effect. Analytical calculations considering a $0.6 \mathrm{~m}$ long pocket made of stainless steel predict a very small resistive wall impedance with a minimal impact on the betatron horizontal tune shift which is limited to less than $1 \cdot 10^{-6}$, well within the stability region defined by the available Landau damping octupoles at LHC [8].

\section{LABORATORY MEASUREMENTS}

The laboratory setup at the Cockcroft Institute comprises a sophisticated mechanical system equipped with micrometer screws, to stretch, move and monitor the relative position of the wire. The absolute position of the wire was estimated at first by moving the wire against the pocket wall and recording the mechanical position when the signal was lost.

We performed measurements in both the time domain and in the frequency domain. A set of measurements based on the reflected signal in time domain (see Fig. 4 in the next section) was used to improve the location of the wire relative to the wall. The impedance of a wire in the vicinity of an infinite perfectly conducting metallic plate is given by [9]:

$$
Z_{c}=60 \operatorname{acosh}\left(\frac{2 b}{d}\right)
$$

05 Beam Dynamics and Electromagnetic Fields
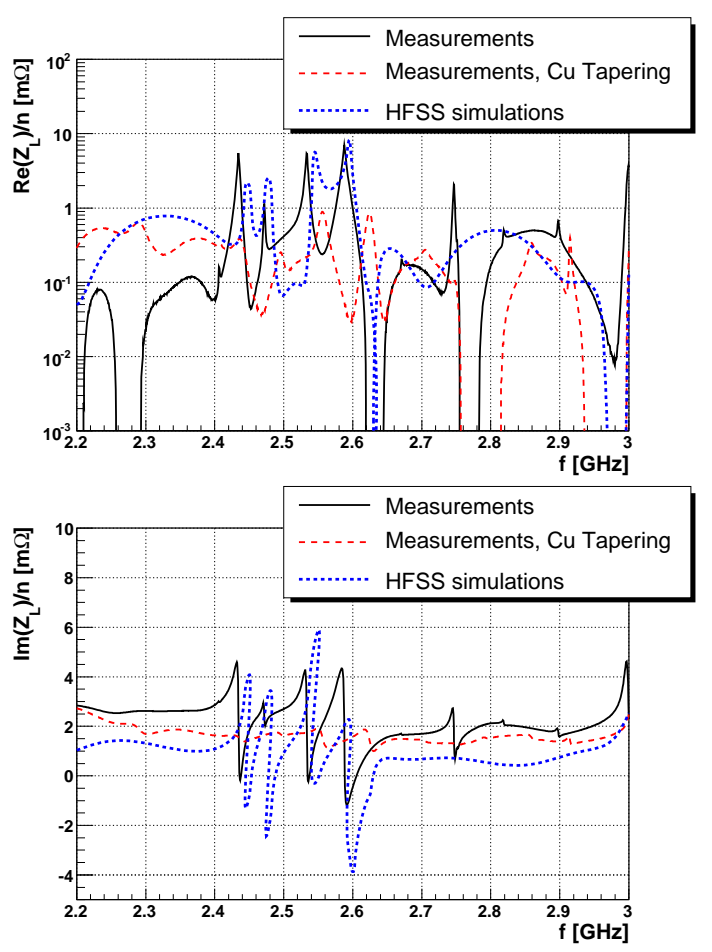

Figure 3: Real and imaginary part of the effective longitudinal impedance.

where $b$ is the distance of the wire from the plate and $d$ the wire diameter. The line impedance is given in terms of the ratio of the incident to the reflected mode amplitude at each:

$$
Z_{c}=Z_{0} \frac{1+S_{i i}}{1-S_{i i}}, \quad S_{i i}=S_{11} \text { or } S_{22}
$$

where $Z_{0}=50 \Omega$ is the cables impedance. Combining Eq. (2) and Eq. (3), one can calculate the distance $b$. This method provided a correction of about $0.4 \mathrm{~mm}$ on the wire position estimation described above.

\section{Longitudinal Impedance Results}

The real and imaginary part of the FP420 effective longitudinal impedance calculated from the measured $S_{21}$ parameter (black solid line) and simulated by HFSS (blue dashed line) are shown in Fig. 3. This plot refers to a wire distance from the pocket wall of $3 \mathrm{~mm}=10 \sigma_{x}$. Measurements and simulations have been carried out for several intermediate distances covering the range from the point at which the pockets are designed to be 'parked' at $x>50 \sigma_{x}$ from the beam to $x=3 \sigma_{x}$, when data is being taken during particle physics events.

The agreement in terms of resonance peaks of the impedance is satisfactory as it lies within $3 \Omega$ in amplitude and few $\mathrm{MHz}$ in frequency. At least one additional resonance appears in the measurements and can be explained by a residual mismatch between the RF source and the DUT, not considered in the simulations.

The FP420 pocket was remeasured after applying a thin 


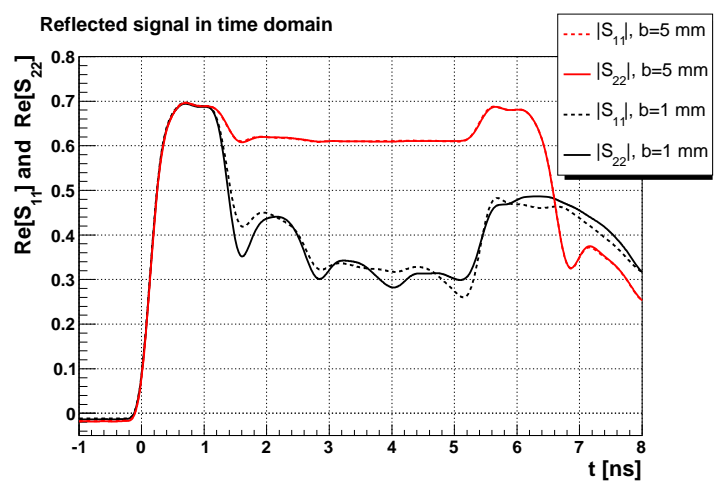

Figure 4: Measurement of the reflected signal for two wire positions.

copper-plated tape at the indentation regions. The tape was placed outside the beam orbit region (i.e. above and below the $500 \mu \mathrm{m}$ thin window), in order to provide a tapered transition of the beam pipe cross section variation. The result is shown by the red solid lines in Fig. 3. After tapering, the longitudinal effective impedance is reduced by an order of magnitude and thus the impedance is limited to no more than $1 \mathrm{~m} \Omega$ over the measured band.

The measurement of the reflection signal in time domain was used to calculate the real part of the longitudinal impedance related to the beam pipe resistivity, disentangled from geometric effects. Looking at Fig. 4, for each wire position, the lower amplitude of the peak at $t_{1} \approx 6 \mathrm{~ns}$ (with respect to the peak at $t_{2} \approx 0.5 \mathrm{~ns}$ ) follows from the losses on the pocket resistive wall. It is thus possible to calculate the quantity

$$
\frac{1}{2} \frac{S_{i i}\left(t_{1}\right)-S_{i i}\left(t_{2}\right)}{S_{i i}\left(t_{1}\right)}=\frac{S_{21}^{D U T}}{S_{21}^{R E F}}
$$

(with $S_{i i}=S_{11}$ or $S_{22}$ ) to be used in Eq. (1) to calculate $Z_{L}$ over the frequency range of the measurements. The result is shown in Fig. 5 and is compared to an analytical formula [10] valid for a beam centered in a cylindrical beam pipe of radius $b$ and with conductivity $\sigma$ :

$$
Z L_{f 1 \rightarrow f 2}=\int_{f 1}^{f 2} \frac{L}{2 \pi b} \sqrt{\frac{2 \pi f Z_{0}}{c \sigma}} d f
$$

The agreement ${ }^{2}$ between measured and analytically calculated values is within $5 \Omega$.

\section{CONCLUSIONS AND OUTLOOK}

The FP420 single pocket geometry has been characterized in terms of longitudinal impedance. Numerical simulations, analytical calculations and laboratory measurements showed consistent results, assessing the small impact on the total LHC impedance budget. Tapering of the beam

\footnotetext{
${ }^{2}$ For completeness, it must be remarked that the present data analysis does not include a correction for the losses in the wire
}

05 Beam Dynamics and Electromagnetic Fields

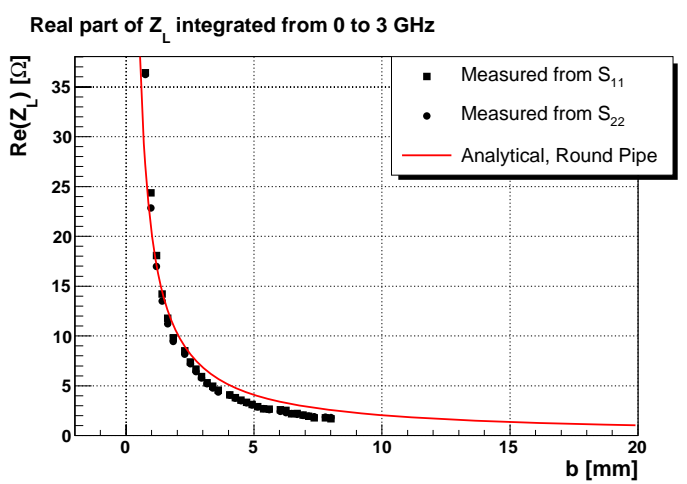

Figure 5: Real part of longitudinal impedance integrated from 0 to $3 \mathrm{GHz}$.

pipe indentations is recommended, although it is not absolutely necessary it does reduce the impedance significantly. Such design modification can be implemented at no cost in terms of forward proton signal over background ratio. Further studies are ongoing in order to determine the characteristic loss factor and beam transverse impedance [11].

\section{ACKNOWLEDGMENTS}

The authors would like to thank W.Bruns, F.Caspers, A.Grudiev, E.Metral and T.Kroyer for their important advice and recommendations on the research and I. Burrows for the help in designing and setting up the laboratory test bench.

\section{REFERENCES}

[1] Cox, B. et al., "FP420 R\&D Proposal", CERN-LHCC2005-025

[2] Caspers, F., "Impedance determination", Handbook of Accelerator Physics and Engineering, World Scientific (1999)

[3] Kroyer, T. et al., "Longitudinal and Transverse Wire Measurements for the Evaluation of Impedance Reduction Measures on the MKE Extraction Kickers", CERN-AB-Note2007-028

[4] Vaccaro, V.G., "Coupling Impedance Measurements", INFN/TC-94/023 (1994)

[5] Ansoft Corporation, URL: http://www.ansoft.com/

[6] Computer Simulation Technology, URL: http://www.cst.com/

[7] GdfidL, URL: http://www.gdfidl.de/

[8] Metral,E., "Overview of Impedance and Single-Beam Instability Mechanisms", CERN-AB-2005-041

[9] Ramo, S., "Fields and Waves in Communication Electronics", John Wiley \& Sons, (1965)

[10] Palumbo, L et al., "Wake Fields and Impedance", CAS - CERN Accelerator School: 5th Advanced Accelerator Physics Course, CERN-REP-95-06-v1

[11] Perry B. Wilson, "Introduction To Wake Fields And Wake Potentials", SLAC-PUB-4547, Jan 1989.

D04 Instabilities - Processes, Impedances, Countermeasures 